\title{
Adolescentes y familia. Aportaciones a la publicidad de telefonía móvil
}

Maite Itoiz López | mitoizl@alumni.unav.es

UNIVERSIDAD DE NAVARRA

Idoia Portilla Manjón I iportilla@unav.es

UNIVERSIDAD DE NAVARRA

Resumen: El teléfono móvil está ampliamente extendido entre los adolescentes y la familia es clave a la hora de facilitarles el acceso a este aparato. En este trabajo el objetivo es analizar el uso de la telefonía móvil que realizan los adolescentes según la familia donde residen, considerando la convivencia o no con hermanos. Los datos proceden de la encuesta del Foro Generaciones Interactivas, seleccionando una muestra de más de 2.000 escolares de 14 y 15 años. A partir de este estudio se realizan recomendaciones para elaborar estrategias publicitarias y de marketing dirigidas a este público.

Palabras clave: adolescentes, familia, telefonía móvil, publicidad

Abstract: The mobile phone is very popular among teenagers and their families are determinant for providing this device. The aim of this study is to analyze the use of mobile phones among teenagers according to their families, considering living or not with siblings. Data comes from the survey of the Foro Generaciones Interactivas, selecting a sample of more than 2000 students 14 and 15 years of age. Recommendations will be made for applying advertising and marketing strategies to this target.

Key words: teenagers, family, mobile phones, advertising 


\section{Telefonía móvil, adolescentes, familia y consumo. Estado de la cuestión}

El éxito de las llamadas tecnologías de la información y la comunicación (TIC) ha creado una nueva sociedad, la denominada "sociedad de la información". Al ser humano se le presentan nuevas habilidades, nuevos conocimientos, nuevas formas de relación. Entre estas tecnologías destaca el teléfono móvil tanto por su penetración como por su crecimiento ${ }^{1}$. Este pequeño dispositivo, que se ha ido sofisticando a lo largo de los años, tiene una amplia difusión gracias a las posibilidades comunicativas que brinda.

Los usuarios más activos del teléfono móvil son, sin duda, los adolescentes (Bringué y Sádaba, 2011; Valor y Sieber, 2004). La disponibilidad de este aparato ha supuesto para ellos una verdadera revolución tecnológica y social (Lorente, 2001 y Lorente, 2002).

\subsection{Adolescentes y telefonía móvil}

Existen varias explicaciones sobre el éxito que ha tenido el teléfono móvil entre este grupo de edad. Ling (2002) expone que la adquisición del teléfono móvil se considera un "ritual de paso", que da entrada a la adolescencia. Fortunati y Magnanelli (2002) hablan de "hermandad virtual", fenómeno que surge entre los jóvenes por compartir sentimientos, emociones y pensamientos (Castellana, Sánchez-Carbonell, Graner y Beranuy, 2007).

El móvil es un instrumento fundamental para el adolescente porque le permite comunicarse con sus iguales (Lenhart et al, 2010). Potencia sus relaciones, le permite mayor intimidad en las conversaciones y le facilita manifestarse de modo más extrovertido (Menor, 2009). Es un gran facilitador de la comunicación (Espinar y González, 2008) y un instrumento socializador (Jiménez y Ramos, 2007; Delgado, Ramos y Jiménez, 2008). Este público utiliza el móvil como medio para organizar las actividades del día a día y para construir un vínculo social definiendo el propio espacio en relación con los otros (Oksman y Rautiainen, 2002).

Esta tecnología también ayuda a los adolescentes a definir su propia identidad (Priede, Segovia, Talón y Villacé, 2005). Se ha ido convirtiendo en un dispositivo que permite expresar la personalidad, es decir, en un símbolo personal (Delgado, Ramos y Jiménez, 2008). Como expresa McLuhan, el móvil se ha transformado, en muy poco tiempo, en una extensión de las extremidades del adolescente (Espinar y González, 2008). Es una prolongación de su cuerpo (Priede, Segovia, Talón y Villacé, 2005).

A través de la personalización del móvil se expresa la identidad de su dueño (Delgado, Ramos y Jiménez, 2008). Es un indicador de estatus social (Muñoz-Rivas y Agustín,

\footnotetext{
${ }^{1}$ En 2011, el 90,7\% de los hogares españoles disponía de teléfono móvil. En 2001, la cifra era 22,7 puntos porcentuales más baja: del 68,0\% (AIMC, 2012, p. 64).
} 
2005) sujeto a los cambios de la moda (Delgado, Ramos y Jiménez, 2008; Phau y Teah, 2009). A través de las carcasas, los tonos o el color van creando un símbolo personal; seña de identidad que define, en muchos casos, la personalidad de los jóvenes del siglo XXI (Jiménez y Ramos, 2007).

Pero además de permitirles comunicarse y definir su identidad, el móvil sirve a los adolescentes para mantener y potenciar ese sentimiento de pertenencia a un determinado grupo (Delgado, Ramos y Jiménez, 2008) expresado, por ejemplo, en el lenguaje utilizado en los mensajes de texto (Castellana, Sánchez-Carbonell, Graner y Beranuy, 2007). Así, van creando su propia cultura hipercoordinando su vida social y construyendo sus redes sociales día a día (Ling and Yttry, 2002). Los móviles permiten un contacto perpetuo (Katz y Aakhus, 2002), un enlace social que se convierte en el modo y la herramienta para expresar su cultura (Caronia, 2008). En definitiva, para los adolescentes el teléfono móvil es parte esencial de su vida social diaria (Ishii, 2006; Kennedy, Smith, Wells y Wellman, 2008).

Pendientes de toda esta situación están las diferentes marcas relacionadas con el negocio de la telefonía móvil. Este aparato es muy relevante para el adolescente y las empresas lo saben (Wilska, 2003). Los adolescentes se han convertido en el foco publicitario de los negocios dedicados a la venta de móviles, así como de otros aparatos como ordenadores o consolas (Espinar y González, 2008). Pero, además de producto de consumo, el móvil puede ser utilizado como canal de comunicación (Caldevilla, 2010). Por tanto, puede ser utilizado para hacer llegar al adolescente mensajes comerciales de otros productos y servicios. Este hecho duplica el interés por conocer el modo en que el adolescente utiliza este apartado.

En cuanto al consumo en general, los adolescentes representan una gran oportunidad (Battle, 2007). Compran productos que les introduzcan en la vida adulta, ya que se encuentran a medio camino entre niños y adultos, e intentan así acercarse a este modo de vida (Jiménez y Ramos, 2007). También atienden a las compras de sus amigos puesto que, cuando una marca se pone de moda, todos los miembros del grupo la desean (Jiménez y Ramos, 2007). Y en todo este mundo del consumo, la familia es clave, por lo que se le prestará especial atención.

\subsection{Los adolescentes digitales y la familia como agente socializador y estructura de consumo}

La familia es el primer agente educador del adolescente (Camps, 2008). Esta institución, junto al grupo de iguales y la escuela, son claves en la socialización de los adolescentes y en el uso que hacen de las nuevas tecnologías (Tolsá, 2012; Malo, 2006). Por lo tanto, la familia es imprescindible en la educación de los adolescentes respecto a estas tecnologías en auge. 
La familia es también una estructura de consumo a la que se han dedicado numerosos estudios (Ekström, 2005). La familia ha dejado de ser una unidad productiva para ser una unidad de consumo (Montoro, 2004). Y en su papel de agente socializador, la familia enseña al adolescente a consumir correctamente, al igual que lo hacen otros como los amigos, la escuela, los medios de comunicación, los comerciantes y las marcas (Moschis y Churchill, 1978; Fan y Li, 2009). Por todo ello, esta institución es grupo de referencia entre los profesionales del marketing, que se mantienen atentos a los cambios en las estructuras y composiciones del hogar (Lee y Beatty, 2002).

Como estructura de consumo, en la familia se toman decisiones de compra en la que influyen niños y adolescentes (Menor, 2009). Su participación es importante tanto en productos para su propio consumo como para el de la familia (Flurry, 2007), pero es si cabe mayor cuando se trata de tecnología. Los adolescentes son grandes usuarios de estos productos y se convierten en prescriptores dentro del entrono familiar (Delgado, Ramos y Jiménez, 2008), debido en muchos casos a que tienen mayores conocimientos técnicos que sus padres (Chordas, p. 108 en Martensen, 2007).

Los mercados deben reconocer y perfilar este segmento de consumo, creando y diseñando promociones dirigidas especialmente a los adolescentes como miembros influyentes en la familia (Lee y Beatty, 2002). En estas acciones, no deben olvidar tampoco la estructura y composición de la unidad familiar y los efectos que pueda tener la misma como agente socializador.

Hoy en día, la situación y estructura de la familia ha cambiado mucho debido a causas sociodemográficas como el retraso a contraer matrimonio, la reducción del número de hijos por mujer debido a su incorporación al mercado de trabajo y al desplazamiento de la maternidad a edades más avanzadas, u otras como el aumento de separaciones y divorcios. Esto da lugar a nuevos modelos de familia como pueden ser los hogares sin hijos, los hogares monoparentales y aquellos que se constituyen sin vínculos familiares.

En el ámbito del consumo destacan los estudios que comparan familias monoparentales frente a familias completas (con padre y madre). Así, se ha demostrado que existen diferencias según se trate de una u otra estructura (Tinson, Nancarrow y Brace, 2008).

El efecto del número de hijos por hogar ha sido menos estudiado. Algunas investigaciones existentes se centran en el estudio de las familias chinas, debido a su limitación a un solo hijo por hogar (Shao y Herbig, 1994; Chan y McNeal, 2003). También hay estudios que comparan familias grandes frente a pequeñas. Estos trabajos han constatado que los niños de familias pequeñas van más a menudo de compras que los niños de familias grandes (Geuens, De Pelsmacker y Mast, 2003). Según Batounis-Ronner, Hunt y Mallalieu (2007), los niños en familias grandes perciben significativamente menos influencia en ciertas decisiones que los niños de familias pequeñas. Por tanto, la presencia de hermanos puede afectar al proceso de consumo y a la percepción que se tenga de la 
influencia en el mismo. En nuestro trabajo, analizaremos si también tiene efecto en aspectos vinculados al consumo y uso del teléfono móvil.

\section{Objetivos y metodología}

El objetivo de este estudio es conocer si existen diferencias significativas en la posesión y el uso de telefonía móvil en familias en donde reside un adolescente solo (hijo único), frente aquellas unidades donde convive con uno o con dos o más hermanos. Concretamente, el estudio analizará diferencias en la posesión de móvil y cómo lo consiguieron (a qué edad, de qué modo y qué tipo de teléfono poseen); en el gasto que conlleva (quién lo paga, tipo de contrato y cuánto gastan al mes); en el uso que hacen del móvil y las personas con quien más se comunican.

A continuación, se presentan los datos utilizados, la hipótesis de trabajo y la técnica estadística empleada para su comprobación.

\subsection{Datos analizados}

Los datos se han tomado del estudio "La generación Interactiva en España" realizado por el Foro Generaciones Interactivas, EducaRed y la Universidad de Navarra². El universo estudiado son alumnos matriculados en primaria, E.S.O. y bachillerato en España, excluyendo Ceuta y Melilla. La muestra válida alcanzada en 2009 es de 12.919 alumnos de 10 a 18 años (Bringué y Sádaba, 2009). La recogida de datos se realizó en las aulas informáticas de los colegios. El diseño y la estructura de la encuesta permiten que no se produzca repetición de respuestas por un mismo usuario así como la protección de la identidad de los participantes (Bringué y Portilla, 2005).

Del total de alumnos entrevistados por el Foro Generaciones Interactivas, se han seleccionado 2.138 alumnos de 14 y 15 años cuya situación familiar es vivir conjuntamente con su padre y su madre.

Se ha limitado la franja de edad a los 14 y 15 años por su generalizada consideración como adolescentes, ya que la acotación por edad de la adolescencia no es unánime (Izco, 2007). Se trata así de evitar disparidades debidas a este aspecto y que puedan verse interrelacionadas con el número de hermanos. Además, en los estudios realizados en el Foro Generaciones Interactivas se observa que la edad es un factor influyente en la conducta de los adolescentes y el teléfono móvil (Bringué y Sádaba, 2009). Al limitar la muestra a personas de 14 y 15 años se busca disponer de un grupo que no presente diferencias importantes entre ellos, tanto en cuanto al uso del móvil como respecto al número de hermanos con los que residen.

\footnotetext{
${ }^{2}$ Ver www.generacionesinteractivas.org.
} 
En cuanto a la selección de familias completas, donde conviven padre, madre e hijos, se ha buscado fundamentalmente evitar la relación entre el número de hermanos y otros aspectos relacionados con la estructura familiar. Como ya hemos dicho, ser o no familia monoparental afecta al consumo (Tinson, Nancarrow y Brace, 2008), por lo que, al seleccionar sólo familias completas, tratamos de evitar este efecto en nuestro análisis.

Finalmente, se han clasificado los casos en tres grupos según el número de hermanos que residen con el adolescente: sin hermanos (vive como hijo único), con un hermano, y con dos o más hermanos (reside en familia numerosa). Del total de 2.138 escolares de la muestra seleccionada, un $14,5 \%$ no reside con hermanos (311 escolares), el 62,3\% reside con un hermano ( 1.332 escolares) y el $23,2 \%$ lo hace con dos hermanos o más (495 escolares).

El cuestionario completo constaba de 126 preguntas. Dado que este trabajo se centra en el uso del teléfono móvil, se han seleccionado las siguientes nueve preguntas que hacen referencia a la disponibilidad y uso de este aparato:

Pregunta 54: ¿Tienes móvil propio?

Pregunta 55: ¿A qué edad tuviste tu primer teléfono móvil?

Pregunta 56: ¿Cómo conseguiste tu primer teléfono móvil?

Pregunta 57: ¿Qué tipo de teléfono tienes actualmente?

Pregunta 58: ¿Quién paga habitualmente el gasto de tu móvil?

Pregunta 59: ¿Qué tipo de medio de pago tienes para el teléfono?

Pregunta 60: ¿Sabes cuánto gastas mensualmente en el teléfono móvil?

Pregunta 61: El móvil te sirve principalmente para...

Pregunta 62: ¿Con qué personas sueles comunicarte más con el móvil?

Estas nueve preguntas están vinculadas a 27 opciones de respuesta que serán estudiadas en el grupo de adolescentes de la muestra elegida.

\subsection{Hipótesis de trabajo}

Nuestro objetivo es comprobar que existen diferencias en la disponibilidad y uso del teléfono móvil según se trate de un adolescente residiendo solo (sin hermanos), residiendo con un hermano, o residiendo con dos hermanos o más (de familia numerosa). Por tanto, nuestra hipótesis de trabajo será la existencia de tales diferencias en los 27 
items del cuestionario del Foro Generaciones Interactivas relacionados con la telefonía móvil.

Tal y como se ha comentado en el apartado anterior, para evitar el efecto de otros factores como la edad y la estructura familiar, se seleccionan adolescentes de 14 a 15 años residentes en familias completas (donde conviven padre, madre e hijos).

Para comprobar si existen diferencias significativas en las respuestas, se aplicará el test de Chi-cuadrado. Esta técnica consiste en contrastar la hipótesis nula de que no hay diferencias significativas entre las respuestas de los adolescentes según el número de hermanos. Si el valor del estadístico es mayor que el valor en tablas de su distribución a un nivel de significación alfa, se rechaza la hipótesis nula y se acepta que existe relación entre las variables (Grande y Abascal, 2009). En este estudio, si se rechaza la hipótesis nula, se confirma nuestra hipótesis de trabajo, pudiendo afirmar que hay diferencias significativas en las respuestas dadas por los adolescentes según el número de hermanos con los que residen.

\section{Resultados y discusión}

Al aplicar el análisis de Chi-cuadrado a las preguntas seleccionadas, observamos que se rechaza la hipótesis nula en diez de las 27 respuestas relacionadas con la posesión y uso del móvil, constatando la existencia de diferencias significativas según el número de hermanos en esos diez aspectos, pero no en el resto (ver tabla 1 ).

No se han detectado diferencias en un elemento importante como es la posesión o no de móvil a esta edad. El teléfono móvil y el consumo del mismo están totalmente extendidos entre los adolescentes y no existen diferencias significativas en función del número de hermanos. El 92,5\% de los adolescentes de 14 y 15 años seleccionados en este estudio tiene teléfono móvil. Esta gran aceptación hace de los adolescentes un perfil comercial especialmente relevante para las compañías de este sector. Desde el punto de vista académico, también está siendo el foco de numerosos estudios (Tolsá, 2012).

Pero aunque la posesión sea similar en el conjunto de adolescentes de 14 y 15 años, las diferencias por número de hermanos con los que residen son relevantes en cuanto a la edad de posesión del primer teléfono móvil. Los adolescentes de familias con menos hijos acceden antes a este aparato (ver tabla 2$)^{3}$.

\footnotetext{
${ }^{3}$ Los porcentajes presentados en las tablas 2 a 5 hacen referencia al total de respuestas válidas dadas por los adolescentes de 14 y 15 años, tengan o no teléfono móvil, ya que son las cifras utilizadas en los cálculos de los estadísticos Chi-Cuadrado.
} 
Tabla 1: Resultados de los tests de Chi-cuadrado.

\begin{tabular}{|c|c|}
\hline Preguntas y opciones de respuesta & Test de Chi-cuadrado \\
\hline 54- ¿Tienes móvil propio? & 0,443 \\
\hline 55- ¿A qué edad tuviste tu primer teléfono móvil? & $0,001 * * *$ \\
\hline 56- ¿Cómo conseguiste tu primer teléfono móvil? & $0,022 * *$ \\
\hline 57- ¿Qué tipo de teléfono tienes actualmente? & $0,004 * * *$ \\
\hline 58- ¿Quién paga habitualmente el gasto de tu móvil? & 0,339 \\
\hline 59- ¿Qué tipo de medio de pago tienes para el teléfono? & 0,272 \\
\hline 60- ¿Sabes cuánto gastas mensualmente en el teléfono móvil? & 0,214 \\
\hline \multicolumn{2}{|l|}{ 61- El móvil te sirve principalmente... } \\
\hline Para hablar & 0,895 \\
\hline Para enviar mensajes & 0,262 \\
\hline Para chatear & 0,107 \\
\hline Para navegar por Internet & 0,292 \\
\hline Para jugar & 0,400 \\
\hline Como reloj o como despertador & 0,298 \\
\hline Para ver fotos y/o vídeos & 0,289 \\
\hline Para hacer fotos & $0,021 * *$ \\
\hline Para grabar vídeos & $0,021 * *$ \\
\hline Como agenda & 0,344 \\
\hline Como calculadora & 0,585 \\
\hline Para escuchar música o la radio & 0,499 \\
\hline Para ver la televisión & $0,002 * * *$ \\
\hline Para descargas (fotos, juegos, fondos, tonos...) & 0,496 \\
\hline \multicolumn{2}{|l|}{ 62- ¿Con qué personas sueles comunicarte más con el móvil? } \\
\hline Con mi madre & $0,050 *$ \\
\hline Con mi padre & $0,051 *$ \\
\hline Con mis hermanos/as & $0,000 * * *$ \\
\hline Con otros familiares & $0,016 * *$ \\
\hline Con los amigos/as & 0,358 \\
\hline Con mi novio/a & 0,487 \\
\hline
\end{tabular}

Significación asintótica (bilateral): *** $\mathrm{p}<0.01$; ** $\mathrm{p}<0.05 ; * \mathrm{p}<0.1$

Atendiendo a estos datos, podemos pensar que el hijo único (sin hermanos) accede antes al mundo de la telefonía móvil. Una razón podría ser que las familias con varios hijos retrasen lo más posible este gasto, ya que su posesión por parte de un hermano puede alentar la demanda del aparato entre el resto de hijos. 
Tabla 2. Edad de posesión del primer móvil. Porcentajes sobre el total de adolescentes.

\begin{tabular}{|c|c|c|c|}
\hline $\begin{array}{c}\text { Número de hermanos } \\
\text { residiendo con el adolescente }\end{array}$ & $\begin{array}{c}\text { Antes de los 12 } \\
\text { años }\end{array}$ & $\begin{array}{c}\text { Después de los } \\
12 \text { años }\end{array}$ & Sin móvil \\
\hline Sin hermanos & $59 \%$ & $35 \%$ & $6 \%$ \\
\hline Un hermano & $49 \%$ & $44 \%$ & $7 \%$ \\
\hline Dos hermanos o más & $50 \%$ & $41 \%$ & $9 \%$ \\
\hline
\end{tabular}

Respecto a cómo consiguieron el primer teléfono móvil, la opción mayoritaria en el conjunto de adolescentes fue obtenerlo como regalo de cumpleaños, Navidad o Comunión $(30,8 \%)$, seguida de "me lo compraron mis padres porque se lo pedí" $(21,1 \%)$. Pero, atendiendo al número de hermanos, los porcentajes con mayores diferencias corresponden a la opción de "me lo compraron mis padres sin pedirlo". Esta respuesta presenta valores significativamente más elevados en familias donde reside sólo un hijo frente a aquellas con varios. Así, existen más adolescentes sin hermanos $(20,6 \%)$ que tienen su primer móvil por iniciativa de sus padres que adolescentes residentes con hermanos (15,9\% entre aquellos con un hermano y $14,2 \%$ entre los de dos o más hermanos). Un motivo podría ser un mayor afán de seguridad y control por parte de los progenitores.

El número de hermanos también parece afectar a si se trata de un móvil de nueva adquisición o de segunda mano. El porcentaje de la opción de segunda mano es más elevado entre los adolescentes con dos o más hermanos (15,6\%), seguido de aquellos con uno (11\%). En cambio, la cifra más baja se da entre adolescentes residiendo sin hermanos (sólo el 8,4\% lo obtiene de segunda mano). Estos datos pueden reflejar la existencia de la herencia del móvil entre hermanos, donde los más pequeños consiguen los teléfonos móviles que abandonan los mayores.

Respecto al gasto que los adolescentes realizan con su teléfono móvil, no existen diferencias significativas en función del número de hermanos. Las respuestas sobre la cantidad gastada, quién paga habitualmente o si se trata de contrato o tarjeta no presentan discrepancias relevantes. Del total de adolescentes de 14 y 15 años, el $52,6 \%$ gasta 10 euros o menos, un $52,5 \%$ tiene el sistema de pago de tarjeta y un $71,4 \%$ reconoce que habitualmente el gasto corre a cargo de sus padres. ${ }^{4}$

En el conjunto de adolescentes, observamos que el móvil tienen numerosos usos (ver tabla 3). Las opciones elegidas por más del 70\% de los adolescentes estudiados son hablar, enviar mensajes y hacer fotos. Además, el móvil sirve con frecuencia de reloj/despertador, cámara de vídeo, calculadora o agenda.

\footnotetext{
${ }^{4} \mathrm{Si}$ analizamos los datos sobre los adolescentes de 14 y 15 años que poseen móvil, cerca del 57\% gasta 10 euros o menos al mes, un porcentaje similar lo tiene de tarjeta y en el $77 \%$ de los casos, el gasto corre a cargo de los padres.
} 
Tabla 3. Usos del móvil. Porcentajes sobre el total de adolescentes.

\begin{tabular}{|l|c|}
\hline El móvil te sirve principalmente... & Porcentaje \\
\hline Para hablar & $88,5 \%$ \\
\hline Para enviar mensajes & $80,5 \%$ \\
\hline Para hacer fotos & $70,5 \%$ \\
\hline Para escuchar música o la radio & $65,1 \%$ \\
\hline Como reloj o como despertador & $59,8 \%$ \\
\hline Para grabar vídeos & $57,2 \%$ \\
\hline Para ver fotos y/o vídeos & $52,6 \%$ \\
\hline Como calculadora & $47,7 \%$ \\
\hline Como agenda & $45,6 \%$ \\
\hline Para jugar & $39,9 \%$ \\
\hline Para descargas (fotos, juegos, fondos, tonos...) & $9,2 \%$ \\
\hline Para navegar por Internet & $7,0 \%$ \\
\hline Para ver la televisión & $6,8 \%$ \\
\hline Para chatear & $3,9 \%$ \\
\hline
\end{tabular}

Atendiendo al número de hermanos, encontramos diferencias significativas a la hora de utilizar el móvil para hacer fotos, grabar vídeos o ver la televisión (ver tabla 4). Estas prestaciones son más utilizadas por adolescentes residiendo solos que por aquellos con hermanos.

Tabla 4. Usos del móvil. Porcentajes sobre el total de adolescentes.

\begin{tabular}{|c|c|c|c|}
\hline $\begin{array}{c}\text { Número de hermanos } \\
\text { residiendo con el adolescente }\end{array}$ & $\begin{array}{c}\text { Hacer } \\
\text { fotos }\end{array}$ & Grabar vídeos & $\begin{array}{c}\text { Ver la } \\
\text { televisión }\end{array}$ \\
\hline Sin hermanos & $74,9 \%$ & $62,7 \%$ & $11,3 \%$ \\
\hline Un hermano & $71,0 \%$ & $57,6 \%$ & $5,7 \%$ \\
\hline Dos hermanos o más & $66,4 \%$ & $52,8 \%$ & $7,1 \%$ \\
\hline
\end{tabular}

Antes hemos visto que, en muchos casos, los adolescentes con hermanos heredaban el móvil. Por tanto, es de esperar que se trate de modelos más antiguos, con menos prestaciones. Por tanto, los hijos únicos dispondrían de móviles más actuales, que ofrecen mejor calidad fotográfica y de grabación en vídeo o que permiten ver televisión, pudiendo explicar que ellos utilicen más estas prestaciones que los adolescentes con hermanos. 
Por último, en las opciones de respuesta de con quién suelen hablar, el $81,1 \%$ de los adolescentes lo utiliza para comunicarse con sus amigos. También destacan en todos los casos las figuras del padre o la madre: un 70,6\% de los adolescentes lo utiliza para hablar con su madre y un 62,4\% declara hablar con su padre. En función del número de hermanos, los hijos únicos destacan a la hora de comunicarse con sus progenitores y con otros familiares (ver tabla 5). Por otra parte, al crecer el número de hermanos, aumenta el porcentaje de adolescentes que se comunica con ellos (ver tabla 5).

Tabla 5. Con quién se comunican con el móvil. Porcentajes sobre el total de adolescentes.

\begin{tabular}{|c|c|c|c|c|}
\hline $\begin{array}{c}\text { Número de hermanos } \\
\text { residiendo con el adolescente }\end{array}$ & $\begin{array}{c}\text { Con mi } \\
\text { madre }\end{array}$ & $\begin{array}{c}\text { Con mi } \\
\text { padre }\end{array}$ & $\begin{array}{c}\text { Con mis } \\
\text { hermanos }\end{array}$ & $\begin{array}{c}\text { Con otros } \\
\text { familiares }\end{array}$ \\
\hline Sin hermanos & $75,2 \%$ & $67,8 \%$ & $13,5 \%$ & $37,6 \%$ \\
\hline Un hermano & $70,8 \%$ & $62,2 \%$ & $31,7 \%$ & $33,9 \%$ \\
\hline Dos hermanos o más & $67,2 \%$ & $59,3 \%$ & $40,9 \%$ & $28,3 \%$ \\
\hline
\end{tabular}

En resumen, se vislumbran algunos campos donde los adolescentes que no conviven con hermanos toman la delantera a los adolescentes que sí lo hacen. Este aspecto puede ser interesante a la hora de forjar las distintas estrategias de marketing, donde este público objetivo puede servir para atraer estas tecnologías al hogar.

\section{Conclusiones}

La presencia del móvil entre los adolescentes es muy elevada y hacen un amplio uso del mismo, tanto para hablar, como para enviar mensajes, hacer fotos y escuchar música o la radio. En la mayoría de los casos el teléfono lo compran sus padres (ya sea por iniciativa del adolescente o de sus progenitores) o bien es regalo de cumpleaños, Navidad o Comunión. Estos aspectos son generales para los adolescentes de 14 y 15 años, independientemente de los hermanos con los que residan.

Sin embargo, entre los adolescentes que no conviven con hermanos, es más habitual que la compra del móvil sea por iniciativa de los padres que propia. Además, este tipo de adolescente accede a su primer teléfono móvil a edades más tempranas y sus móviles suelen ser de nueva compra. Por el contrario, los adolescentes residiendo con hermanos tienen, con mayor frecuencia, teléfonos móviles de segunda mano. Esta idea puede ser explotada por las compañías a la hora de crear estrategias de marketing. Familias con varios hijos pueden ser destinatarios de promociones para cambiar de teléfono móvil con mayor frecuencia, de modo que se facilite el traspaso de aparatos entre miembros. Por otra parte, el crecimiento de las familias con un hijo puede suponer una creciente demanda temprana de teléfonos móviles. Las compañías deberían estar atentas a tal circunstancia. 
Otro aspecto destacado en esta investigación es que los adolescentes que no residen con hermanos parecen disponer de teléfonos móviles de más alta gama que los adolescentes de familias más grandes. Este aspecto puede interesar a las compañías, ya que estos adolescentes pueden ser prescriptores y líderes entre sus iguales y en el núcleo familiar.

La ausencia de hermanos se relaciona con un mayor uso del móvil para hablar con los padres o con familiares distintos de los hermanos. Sin embargo, con quien más hablan todos los adolescentes son con sus amigos, independientemente de los hermanos con los que convivan. Esto corrobora la relevancia del teléfono móvil como instrumento socializador.

Aspectos como la cantidad gastada mensualmente en el teléfono móvil, el sistema de pago (tarjeta o contrato) y quién se hace cargo del gasto, no han mostrado relación con el número de hermanos. Aunque podría esperarse un mayor gasto por hijo en el caso de adolescentes que no conviven con hermanos, no existen diferencias estadísticamente significativas que corroboren este supuesto. La disponibilidad del móvil o el gasto que se realiza no es mayor entre quienes pueden ser hijos únicos respecto a los adolescentes de familias numerosas.

En conclusión, existen características de la familia como el número de hijos, que pueden condicionar el consumo de telefonía móvil de los adolescentes. Las empresas de telefonía y las que utilicen este aparato como soporte publicitario deben estar atentas a esta relación entre adolescentes, familia y telefonía móvil. Se trata, además, de un proceso en continuo cambio y que, por tanto, precisará investigaciones que completen y amplíen este trabajo. 


\section{REFERENCIAS BIBLIOGRÁFICAS}

AIMC (2012): Marco general de los medios en España. AIMC, Madrid.

Batounis-Ronner, C., Hunt, J.B. y Mallalieu, L. (2007): "Sibling effects on preteen children's perceived influence in purchase decisions" en Young Consumers, 8 (4); 231-243.

BATTLE, R. (coord.) (2007): "Adolescents i entorn: connexió i compromís" en $V$ Jornades d'Educació en Valors: constrint la ciutadania des de l'educació. Vic.

BRINGUÉ, X. y Portilla, I. (2005): "Encuestas en Internet: Aplicación de las TIC entre los escolares ", en Jaime Andreu Abela et al (eds.), III Congreso de Metodología de Encuestas. 15, 16 y 17 de Septiembre de 2004. Universidad de Granada, Granada, 335-339.

BRINGUÉ, X. y SÁDABA, C. (2011): La Generación Interactiva en Madrid. Madrid. Generaciones Interactivas-Fundación Telefónica. Disponible en: http://www.generacionesinteractivas.org/upload/libros/La\%20Generaci\% C3\%B3n\%20Interactiva\%20en\%20Madrid.pdf

BRINGUÉ, X. y SÁDABA, C. (2009): La generación interactiva en España. Niños y adolescentes ante las pantallas. Madrid, Ariel. Disponible en: http://www.generacionesinteractivas.org/upload/libros/LaGeneraci\%C3\%B3n-Interactiva-en-Espa\%C3\%B1a.pdf

CAMPS, V. (2008): "El valor de la familia en la sociedad del conocimiento". Ponencia presentada en Familias y globalización. Madrid.

Caldevilla, David (2010): "Nuevas lecturas del concepto de publicidad a partir de las TIC", Questiones publicitarias: revista internacional de comunicación y publicidad, 15; 35-51

CARONIA, L. (2008): "Talk-in-interaction and everyday construction of culture: the case of mobile phone communication between parents and children". Ponencia presentada en la reunión anual de la International Communication Association, TBA, Montreal, Quebec, Canadá.

Castellana, M.; Sánchez-Carbonell, X.; Graner, C. y Beranuy, M. (2007): "El adolescente ante las tecnologías de la información y la comunicación: internet, móvil y videojuegos", en Papeles del Psicólogo, 28; 196-204.

CHAN, K. Y MCNEAL, J. U. (2003): "Parent-child communications about consumption and advertising in China" en Journal of Consumer Marketing, 20 (4); 317-334. 
Delgado, M. T.; Ramos, M. y Jiménez, G. (2008): "La publicidad de móviles dirigida a adolescentes: El teléfono móvil como reflejo de la personalidad de los jóvenes" en C. Etayo et al (eds.), Los jóvenes y el nuevo escenario de la comunicación. Actas del XXI Congreso Internacional de Comunicación, EUNSA, Pamplona, 475-488. Disponible en: www.unav.es/fcom/cicom/2006/docscicom/4_RAMOS.pdf

EKSTRÖM, K.M. (2005): "Rethinking family consumption-Tracking New research Perspectives" en Advances in Consumer research 32; 493-505.

ESPINAR, E. y GonZÁLEZ, M. J. (2008): "Jóvenes conectados. Las experiencias de los jóvenes con las nuevas tecnologías" en RES. Revista Española de Sociología, 9; 109-122.

FAN, Y. y LI, Y. (2009): "Children's buying behaviour in China. A study of their information sources" en Marketing Intelligence and Planning, 27; 1-31.

FLURRY, L. A. (2007): "Children's influence in family decision-making: Examining the impact of the changing American family" en Journal of Business Research, 60; 322-330.

Fortunati, L. y Magnanelli, A. M. (2002): "El teléfono móvil de los jóvenes" en Estudios de Juventud, 57; 59-78.

Geuens, M, De Pelsmacker, P y MASt, G (2003): "How family structure affects parent-child communication about consumption" en Young Consumers: Insight and Ideas for Responsible Marketers, Vol. 4 Iss: 2, 57-62.

GRANDE, I y ABASCAL, E. (2009): Fundamentos y técnicas de investigación comercial. Madrid. Esic Editorial.

ISHII, K. (2006): "Implications of mobility: The uses of personal communication media in everyday life", en Journal of Communication, 56, 346-65.

IzCO, E. (2007): "Los adolescentes en la planificación de medios. Segmentación y conocimiento del target". Tesis doctoral premiada con accésit por Injuve. Disponible en: http://www.injuve.es/contenidos.item.action? $\underline{i d=648096574 \& \text { menuId }=400352110}$

JimÉnEz, G. y RAMOS, M. (2007): "Jóvenes y móviles. Estrategias de los operadores de telefonía en España", en Comunicar, 29; 121-128.

KATZ, J. E. Y AAKhuS, M. A. (2002): Perpetual Contact: Mobile Communication, Private Talk, Public Performance. Cambridge MA. Cambridge University Press. 
Kennedy, T. K. M., Smith, A., Wells, A. T. y Wellman, B. (2008): "Networked families. Parents and spouses are using the internet and cell phones to create a "new connectedness" that builds on remote connections and shared internet experiences. The Pew Internet and American life proyect. Disponible

en: http://pewinternet.org/ /media/Files/Reports/2008/PIP Networked Fami ly.pdf.pdf

LEE, C.K.C. y BEATTY, S.E. (2002): "Family structure and influence in family decision making" en Journal of Consumer Marketing 19 (1); 24-41.

Lenhart, A., Ling, R., CampbelL, S. \& Purcell, K. (2010). Teens and Mobile Phones: Text messaging explodes as teens embrace it as the centerpiece of their communication strategies with friends. Washington, D. C., Pew Internet \& American Life Project. Disponible en: http://pewinternet.org/ /media//Files/Reports/2010/PIP-Teens-andMobile-2010-with-topline.pdf

LING, R. (2002): "Chicas adolescentes y jóvenes adultos varones: dos subculturas de teléfono móvil" en Estudios de Juventud, 57; 33-46.

LING, R. y YTTRI, B. (2002): "Hyper-coordination via mobile phones in Norway", en Perpetual Contact: Mobile Communication, Private Talk, Public Performance, eds. James E. Katz and Mark Aakhus Cambridge: Cambridge University Press; 139-169.

LORENTE, S. (2001): "Comunicación, relaciones primarias y teléfono móvil". Ponencia presentada en el Congreso de Sociología. Salamanca.

LORENTE, S (2002): "Juventud y teléfonos móviles: Algo más que una moda", en Revista de Estudios de Juventud, 57; 9-24.

MaLo, S. (2006): "Impacto del teléfono móvil en la vida de los adolescentes entre 12 y 16 años", en Comunicar, 27; 105-112.

MARTENSEN, A. (2007): "Tweens' satisfaction and brand loyalty in the mobile phone market", en Young Consumers, 8; 108-116.

MCNEAL, J.U. (1992): Kids as customers: a handbook of marketing to children. New York. Lexington Books.

MenOR, J. J. (2009): Adolescentes y jóvenes en la red: Factores de oportunidad. Madrid. Instituto de la Juventud.

Montoro, R. (2004): "La familia en su evolución hacia el siglo XXI". Ponencia en II Congreso La familia en la sociedad del siglo XXI. Madrid. FAD.

MoschIS, George P. y CHURCHILL, Gilbert A. Jr. (1978): "Consumer Socialization: A Theoretical and Empirical Analysis", en Journal of Marketing Research, v. 15 , n. 4 , pp. 599-609. 
Muñoz-Rivas, M. J. y Agustín, S. (2005): "La adicción al teléfono móvil" en Psicología Conductual, 13; 481-493.

OKSMAN, V y RAUTIAINen, P. (2002): "Toda mi vida en la palma de mi mano: la comunicación móvil en la vida diaria de los niños y adolescentes de Finlandia" en Estudios de Juventud, 57; 25-32.

PHAU, I. Y TEAH, M. (2009): "Young consumers' motives for using SMS and perceptions towards SMS advertising", en Direct Marketing: An International Journal, 3; 97-108.

Priede, T.; Segovia, M.; Talón, P. y Villacé, M. T. (2005): "Identificación de los factores clave en el comportamiento de los jóvenes en el sector de la telefonía móvil". Ponencia presentada en XII Asamblea General ALAFEC. Lima.

SHAO, A.T. y HERBig, P. (1994): "Marketing implications of China's 'Little emperors'" en Review of Business, 16; 1-5.

Tinson, J., Nancarrow, C. y Brace, I. (2008): "Purchase decision making and the increasing significance of family types", en Journal of Consumer Marketing, 25; 45-56.

TolsÁ, J. (2012): Los menores y el mercado de las pantallas: una propuesta de conocimiento integrado. Madrid, Colección Generaciones Interactivas Fundación Telefónica. Disponible en: http://www. generacionesinteractivas.org/upload/libros/Los\%20menores\%20y\%20el \%20mercado\%20de\%20las\%20pantallas.pdf

VAloR, J. Y Sieber, S. (2004): "Uso y actitud de los jóvenes hacia internet y la telefonía móvil". Estudio realizado por PWC y IESE.

WILSKA, T. (2003): "Mobile phone use as part of young people's consumption styles", en Journal of Consumer Policy, 26; 441-463.

[Recibido: 23 de mayo de 2011. Aceptado (con solicitud de cambios): 14 de mayo de 2012. Recepción del artículo corregido: 15 de mayo de 2012]. 